\title{
Resultados Sobre la Práctica del Diseño Asociado con el Trabajo Colaborativo y el Construccionismo en una Comunidad de Guarulhos, Brasil. \\ Findings on the Design Practice Associated with the Collaborative Working and Constructionism in a Community of Guarulhos, Brazil
}

\author{
> Alex Garcia Smith Angelo \\ University of São Paulo, Brazil \\ alexgarcia@usp.br \\ > Elizabeth Romani \\ University of São Paulo, Brazil \\ bethromani@usp.br
}

\author{
> Juliana Harrison Henno \\ University of São Paulo, Brazil \\ julianaharrison@usp.br \\ > Milton Villegas Lemus \\ Costa Rica Institute of Technology, Costa Rica \\ mvilem@usa.net
}

\begin{abstract}
This article has a purpose to introduce a methodology to learn the product design principles, applied with the children of a neighborhood in the City of Guarulhos, State of São Paulo. This project is developed through a workshop organized with the collaboration of the Unified Educational Center, two companies and a digital inclusion program of the Municipality of Guarulhos. In order to provide an understanding on the development stages of a product from its design through its completion, the workshop allowed the participants to learn together and provided the access to a simplified design procedure.
\end{abstract}

Keywords: Design, Digital Manufacturing, Society, Technology Learning, Collaborative Network

\section{Introduction}

La interacción entre el aprendizaje y la tecnología es un reto para la sociedad del siglo XXI. En Brasil, se puede inferir la ausencia de un programa colectivo, público y democrático que tome ventaja de las tecnologías digitales y así ayude al desarrollo educativo y social de las comunidades locales. Este punto de vista limita el potencial de las tecnologías que, cuando se combinan con otras áreas del conocimiento podrían contribuir de forma más efectiva al desarrollo comunitario local. Más específicamente, los actuales programas de formación, ya sean en la educación pública o en la inclusión digital explotan poco el ejercicio de conceptos de dibujo proyectivo o diseño y sus relaciones con las nuevas tecnologías.

Ante este escenario, el Departamento de Informática y Telecomunicaciones del ayuntamiento de Guarulhos coordina el Fab Social, que es un intento de proporcionar una experiencia de conceptos de diseńo en forma de talleres para niños y adolescentes. Las actividades desarrolladas por Fab Social por lo general ocurren en los Centros de Educación Unificada (CEU) de la ciudad de Guarulhos. El propósito del CEU es vincular las prácticas educativas, culturales y de ocio en un espacio abierto a las comunidades circundantes.

En este artículo se describe la experiencia de una actividad llevada a cabo por el Fab Social en CEU Rosa de Francia. La concepción de este taller tuvo su origen a partir del hallazgo de la relevancia de modelos de actividades que posean objetivos similares defendidas por Cross (1982), es decir, que el pueblo que participa de una actividad de diseño puede contribuir en el diseño y construcción de su propio entorno. Tal acción, según Cross, promovería una actitud crítica hacia el diseño de productos, servicios y el desarrollo de habilidades de síntesis de un problema propio de la actividad de los diseñadores. La acción entre el público y la práctica del diseño, enfatiza aún más el papel del diseñador como mediador entre la sociedad y la tecnología tal como se quiere demostrar en este artículo.

Este enfoque también se pude hallar en Bonsiepe (2011), que establece que con la llegada de la tecnología informática y el concepto de interfaz permite apreciar el diseño como un mediador entre el usuario y el producto. Esa premisa está de acuerdo con la de Maldonado (2009 pg.191), en la que “(...) los avances en las tecnologías están despertando una creatividad técnico-científica que permite la manipulación de proyectos sin precedentes (...) la tecnología moderna traerá un mundo dominado por objetos y procesos técnicos en que la proyectualidad estará omnipresente (...) “. En referencia a esta etapa tecnológica, Gershenfeld (2012) se refiere a la revolución de la fabricación digital, como el momento en el que las personas traspasan los límites del entorno virtual y diseñan y producen objetos en el mundo real. Como complemento de este punto de vista, otros autores como Resnick et al. (2001) confirman que no sólo el acceso a la tecnología es lo principal, sino también las nuevas formas de interacción social y las nuevas áreas de conocimiento que surgen de estas interacciones entre la tecnología y la gente, en especial los que viven en zonas remotas.

Como antecedente de la actividad Fab Social se tiene una experiencia pionera que tuvo lugar en Brasil, en São Paulo entre los años 2001 y 2004, con el proyecto $A$ cidade que a gente quer que tuvo como organizadores el Secretaria de Educação de Sáo Paulo, el 
MIT - Media Lab y el Laboratório de Sistemas Integráveis da Escola Politécnica da Universidade de São Paulo. El proyecto se realizó en las escuelas públicas de São Paulo y los participantes diseñaban y construian modelos de cómo les gustaría mejorar la calidad de vida en sus ciudades usando algunos elementos como: una placa electronica, materiales de bajo costo y reuso.

Algunas de estas ideas que ya fueron exploradas, específicamente en el año 2002, se pueden encontrar de las experiencias del profesor Mikhak en el Media Lab y su grupo Grassroots Invention Group, en particular con el proyecto sobre fabricación personal Fab Lab, cuando estudiaban los efectos de la revolución de fabricación personal y su potencial. En especial se efocaban más allá de las tecnologías de las herramientas, también estudiaban cómo esas herramientas facilitaban las relaciones que se pueden dar a las fases de diseño, programación, recolección de datos, análisis.

En el marco de los estudios mencionados, el profesor Mikhak desarrolló el proyecto Red para Aprender Independencia, (Learning Independence Network). Una propuesta para la verdadera transferencia de tecnología. El piloto de esta propuesta de desarrollo se implantó en Costa Rica en el 2002..

Como parte del proyecto Red para Aprender Independencia se desarrolló el Laboratorio LuTec, en el Centro de Investigaciones en Computación del Instituto Tecnológico de Costa Rica (Costa Rica FabLab). Un laboratorio cuyo trabajo se centra en promover el aprendizaje de los conceptos de ciencia y tecnología en niños de zonas rurales en Costa Rica. El fundamento pedagógico de este laboratorio tiene sus raíces tanto en el construccionismo de Papert, en su enfoque de cómo el aprendizaje es más rápido y significativo cuando es contextual como en la aplicación de la fabricación digital en el proceso de construcción de prototipos que resuelvan problemas específicos de las comunidades de Mikhak.

Uno de los proyectos de laboratorio LuTec es el Kit de Robótica Costarricense: Krotic. Este proyecto consiste en una serie de módulos que permiten la construcción de un robot de propósito educativo de bajo costo (menos de US\$75). Los módulos con los que se construye el robot se pueden usar individualmente o usar algunos de ellos, no necesariamente todos, para que los chicos y chicas construyan sus propios prototipos de proyectos. Muchos de estos prototipos no tienen que ver con el robot totalmente ensamblado.

Fue en el marco de trabajo del laboratorio LuTec que se decidió, en conjunto con investigadores de la Municipalidad de Guarulhos en 2011, evaluar la realización de talleres usando videoconferencia para contactar estudiantes de Costa Rica con chicos y chicas en Brasil. También el próposito de dicha actividad fue compartir ideas de los fudamentos pedagógicos de estas actividades.

Las experiencias y trabajos relacionados anteriormente contribuyeron en la definición de una estrategia novedosa, para el uso de la fabricación digital en el aprendizaje de conceptos básicos para el Diseño. Estos conceptos se refieren al desarrollo del lenguaje gráfico del niño con la máquinas y la coordinación para la construcción de prototipos de producto. Para los autores dichos conceptos básicos conforman el Diseño. No están cargados de tecnicismos propios del diseño profesional que pueden desviar la atención del aprendizaje del proceso de la construcción rápida del prototipo. Se debe aclarar que se hace incapie en el proceso, más no tanto el prototipo en si, por lo tanto no era problema si los chicos no terminaban el proyecto. No se conocen trabajos previos alrededor del uso de la fabricación personal, el aprendizaje construccionista y el trabajo colaborativo aplicados al aprendizaje de las etapas de los conceptos básicos del Diseño. Por eso, el intento de los autores de este artículo fue en establecer nuevas metodologías para se descubrir nuevas opciones de trabajo.

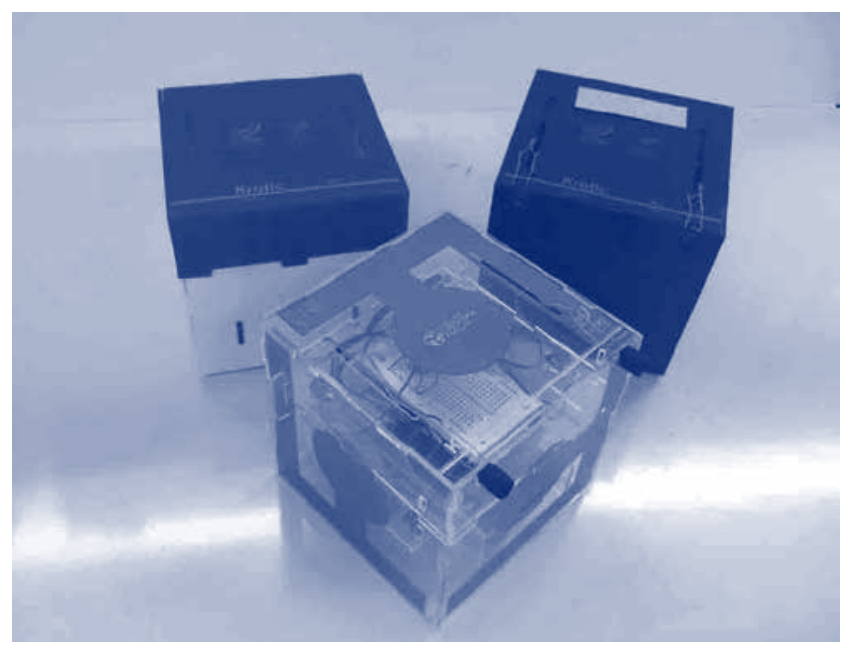

Figura 1: Última versión Kits de robótica Krotic de LuTec.

\section{Metodologia}

Un taller piloto se llevó a cabo en el periodo comprendido entre el 26 agosto y 6 septiembre de 2013 y contó con la presencia de diez participantes con la coordinacion del arquitecto del ayuntamiento Alex Garcia . Todos los participantes eran usuarios del CEU (Centro de Educacíon Unificado) Rosa de Francia, y la mayoría de ellos de edades comprendidas entre 8-17 años. La metodología aplicada en este taller induce la actividad de participar en la encuesta y el diagnóstico del problema, solución propuesta y ejecución de proyectos. La actividad se dió en cuatro pasos, como se describe a continuación.

\section{Paso 1: Recolección, sistematización de datos y diagnóstico}

En esta etapa del taller, se alentó a los participantes a realizar un estudio de necesidades de muebles del CEU Rosa de Francia. Los estudiantes se dividieron tanto en parejas como en tríos. Estos grupos examinaron la recinto para observar cuáles eran estas necesidades de muebles. La investigación de campo se llevó a cabo por medio de fotos digitales, videos, notas y dibujos a mano alzada. A partir de la encuesta llevada a cabo por los grupos, éstos presentaron a los demás participantes sus impresiones sobre los datos recogidos. Con base en estos resultados, se encontraron las siguientes deficiencias: falta de los espejos de los baños, falta de el tablero de puntuación en la pista polideportiva, zapateros, bebederos, tablero de anuncios, ganchos para mochilas, falta de estacionamiento para bicicletas, falta de rampas de acceso y pocos basureros. Para la presentación en el salón de clases, los participantes utilizaron el software libre Impress. Después de dicha presentación, todos los diagnósticos 
realizados por cada grupo, los participantes se votaron por escoger la necesidad más relevante. Es importante destacar que el arquitecto a cargo de la actividad, indujo a los grupos a que se seleccionara un objeto viable para su implementación. Al final la propuesta elegida fue: fabricar cuatro contenedores destinados a recoger los materiales reciclables.

\section{Paso 2 : Diseño}

Una vez definido el problema, cada uno de los grupos participantes realizó su propia encuesta sobre el objeto elegido, se usaron también búsquedas en Internet, para ampliar el repertorio de referencias de los participantes. Esto les permitió a los grupos en la etapa de diseño, proponer nuevas formas al objeto e incluso funciones adicionales a los basureros. En esta etapa, los participantes diseñaron a mano alzada, con el fin de lograr una mayor libertad expresiva para crear formas y no estár restringido al conocimiento técnico individual. Durante este proceso de definición de la forma, los grupos elegieron ciertos parámetros proyectivos para las funciones de sus basureros, algunos de los parámetros establecidos fueron: facilidad de movimiento, altura ideal para los chicos y forma creativa para fomentar su uso. Con base en los criterios, los participantes determinaron el tamaño ideal, el uso de ruedas, la adecuación de la entrada y salida de los residuos en relación con la forma y la posible interacción con la persona que tiró a la basura. Después de definir las fuciones, se motivó a los grupos a pensar en la apariencia del producto. De forma colectiva se elegieron un personajes de ficticios buscados por los chicos en Internet, con aspecto de híbrido de mascotas y animales. Con el dibujo a mano alzada de los personajes, los grupos usaron los principios de un "diseñador", es decir, conceptos de diseño de la forma y de la función. Podemos demostrar esto en la figura 2 en la que los personajes ya incorporan los parámetros definidos por los grupos por ejemplo ruedas, ganchos y las bocas.

Debido a que se trabajó en la apariencia de la cara frontal, fue necesario establecer la forma de cómo iban a entrar los residuos. Se puede notar aquí, otro aspecto importante del diseño enfrentado por los chicos. Al contrario de los basureros convencionales, en los que la abertura está en la parte superior, se optó por un orificio de entrada en la posición de la boca de la mascota, situado en la parte delantera. Otra hecho interesante fue la apropiación espontánea de cada proyecto, prueba de esto fue que cada basurero fue bautizado por los niños. Se pueden apreciar en la foto los dibujos de los basureros: Sammy, Sapinho, Luvinha, Gelatina. La parte frontal de cada uno dibujos de basura fueron transferidos a la computadora, el arquitecto a cargo digitalmente transfirió los diseńos finales, para proceder a fabricarlos.

\section{Paso 3: Producción}

Los archivos digitales transcritos en el paso anterior, se lograron utilizando el software Rhinoceros 3D, con el fin de hacer el archivo compatible con el enrutador CNC. Esta es una máquina de fresado de control numérico que puede tambien hacer cortes en materia prima como el caso de MDF $30 \mathrm{~mm}$, de acuerdo con la forma en el archivo digital que se le envíe. Una compañia local de ventas de máquinas CNC, llamada TecnoFlexo colaboró en el taller con los cortes de las caras frontales de los basureros. En paralelo a las caras frontales, la estructura principal de la basura fue diseñada y fabricada por una carpintería local llamada Maranello fue dirigido por carpintería de con el mismo MDF 30mm pero de color blanco. Se seleccionó el contenedor con la altura de boca más alta para incorporarle sonido. Para el caso del contenedor seleccionado se usó un reproductor de MP3 empotrado, de forma tal que cuando se mueve la compuerta para botar algo se activa el sonido.

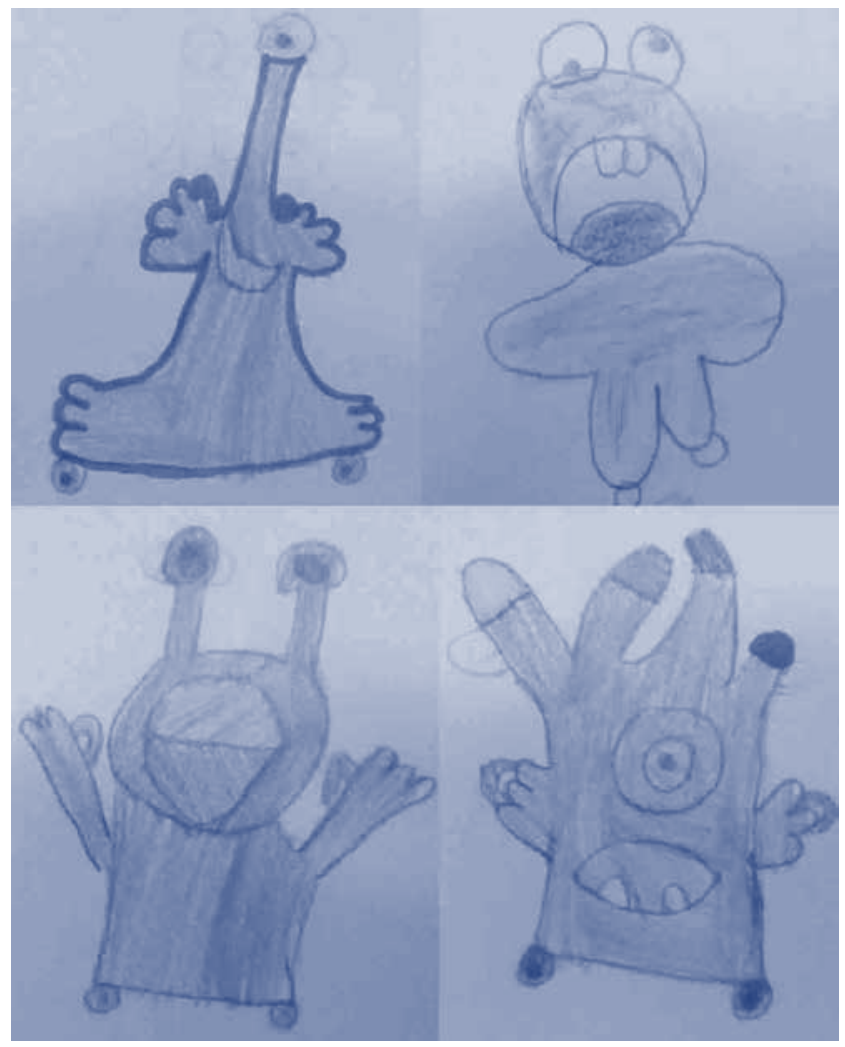

Figura 2: Dibujos de las caras frontales.

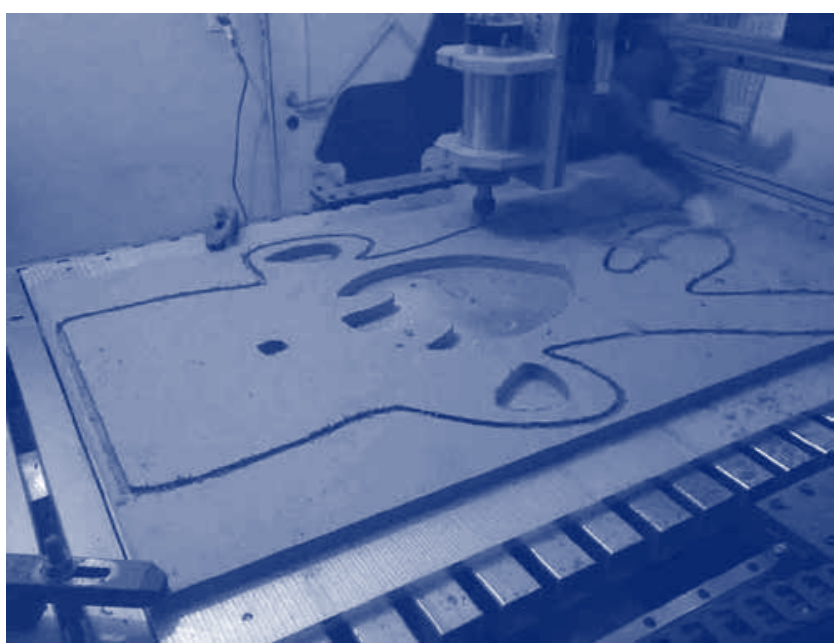

Figura 3: Producción de la fresadora CNC. La carpintería. 


\section{Paso 4: Acabado}

Debido a un cambio de coordinador del CEU, hubo una discontinuidad en el proyecto, lo que a su vez provocó que el grupo de chicos que participó en el inicio del proyecto no coincidiera con el grupo que dió el acabado final a los basureros. El acabado de la basurero, se llevó a cabo en un acto de aniversario del CEU Rosa de Francia el 15 de marzo 2014. Dentro de las actividades del día, les fue propuesto a los chicos de la comunidad, la posibilidad de pintar a su gusto los basureros, de hecho algunos fueron pintados varias veces por diferentes grupos de chicos.

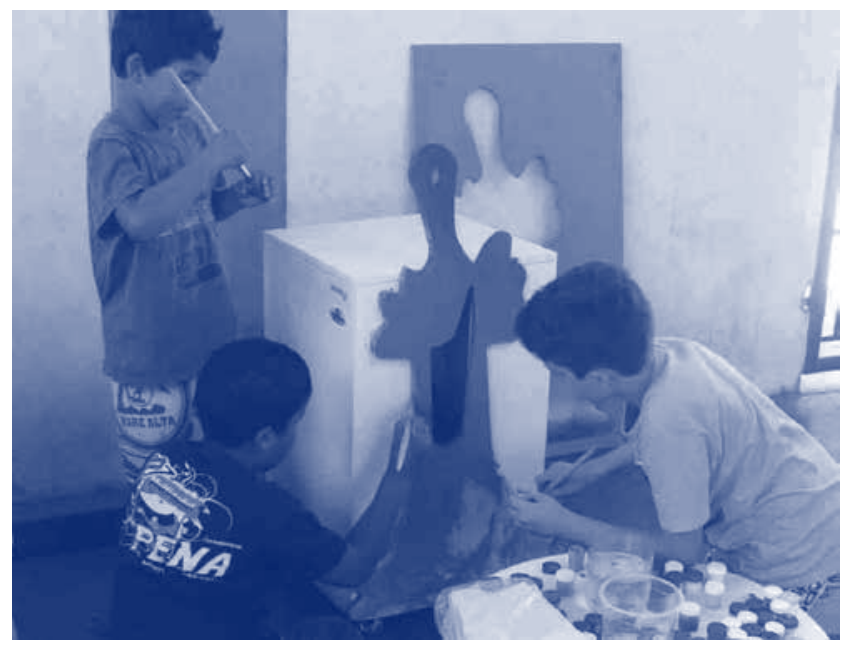

Figura 4: La etapa de acabado.

\section{Resultados}

Del primer paso, recolección, sistematización de datos y diagnóstico se observó que los niños encontraron en instrumentos, como fotografia digital y grabadoras video herramientas que se podían usar, en conjunto con el dibujo a mano alzada y anotaciones, para llevar a cabo la tarea de análisis. Dieron a dichos instrumentos una aplicación más formal a instrumentos asociados con el esparcimiento.

Como producto del segundo paso, los participantes trabajaron en conjunto con el motor de búsqueda Google, para desarrollar sus propuestas conjugadas con el dibujo a mano alzada para realizar sus propias propuestas de diseńo. Esto es, integraron elementos del mundo digital con aportes propios para obtener las nuevas propuestas. La búsqueda de referencias es aspecto importante en el diseño aunque algunas veces controversial.

En el tercer paso (la producción) se dió lo siguiente: dado que las empresas patrocinadoras facilitaron la tecnología, fue claro comprobar que una actividad del diseñador o arquitecto está en servir de interfaz entre ellos, es decir la tecnología- sociedad. En el caso de este proyecto las empresas Tecnoflexo y Maranello (la tecnología) con la comunidad CEU Rosa de Francia (la sociedad).

En el paso 4, el acabado, se obtuvo la apreciación de que la cultura imaginaria de los chicos, permitió una gran aceptación de otros chicos a involucrarse con un proyecto que ellos no habían iniciado. Hubo una comunicación no verbal sobre la intención de las caras delante de cada basurero, que los otros niños pudieron interpretar, en muchos casos pintandolos de forma reiterada varias veces durante el día de la actividad.

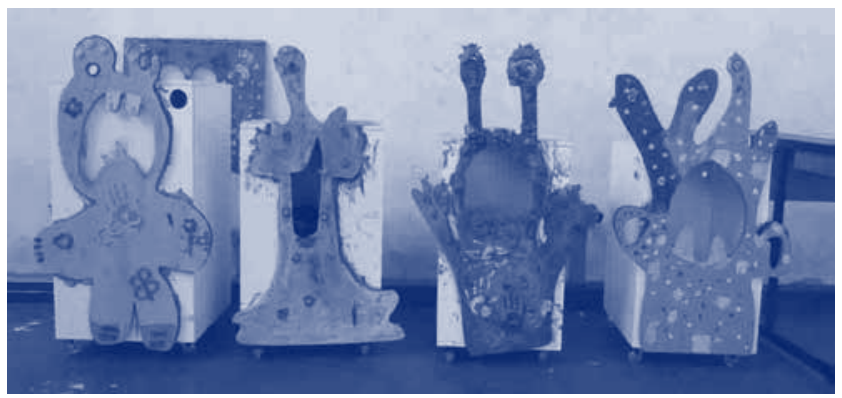

Figura 5: Basureros acabados.

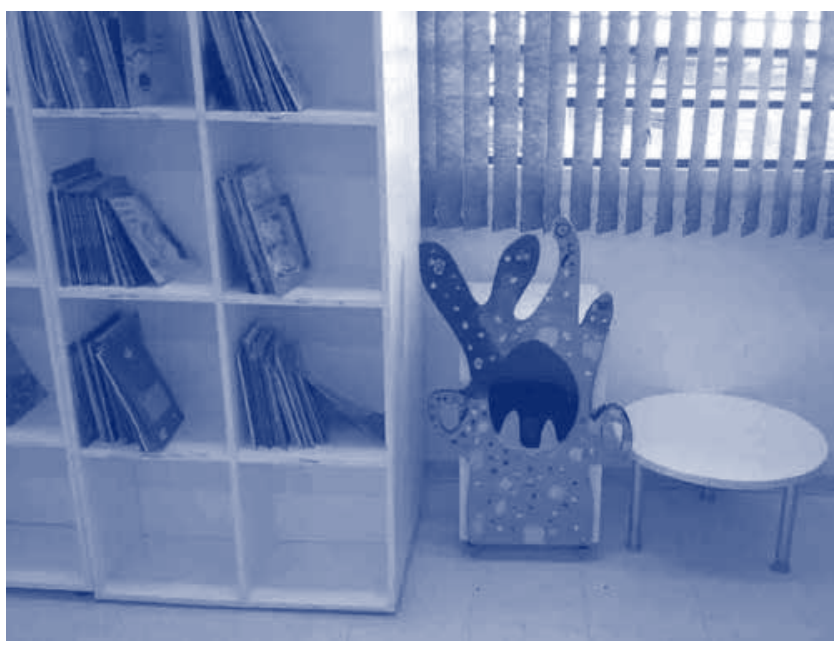

Figura 6: Basurero .

\section{Conclusión}

Se llegó a la conclusión de que una iniciativa de actividad de diseńo con personas no especializadas, puede promover el aprendizaje de los siguientes conceptos: recolección, sistematización de datos, diagnóstico, diseño, producción y acabado. Esos conceptos, de acuerdo con los autores, son indispensables para la educacion general. Por lo tanto, este artículo contribuye al debate de la actividad de diseño como interfaz, que excede a todo conocimiento de las áreas académicas y alcanzan las comunidades y su desarrollo social.

La evaluación de la actividad descrita en el presente artículo arrojó resultados positivos, pero también mostró otros aspectos que mejorar. Uno de los aspectos a mejorar fue que los autores no midieron cuantitativamente, el impacto sobre el aprendizaje, en los participantes de la actividad.

Uno de los resultados de este proceso fue que se reflexionó sobre dos metodologías posibles de trabajo. Una primera en la que los participantes tienen acceso a todas las actividades o pasos, desde la recolección, hasta el acabado y otra metodología que agrupa los participantes a trabajen o desarrollen una actividad cada y posteriormente todas las partes se integran. 
En el proceso de preparación y desarrollo de la actividad en conjunto de comunidades culturales cercanas permitió establecer una red de conocimiento. Los autores esperan continuar con el trabajo en colaboración, estableciendo la participación de cada uno en las diferentes etapas de diseño y aplicando instrumentos para mejorar la evaluación de resultados.

\section{References}

Angelo, A., Campos. P, \& Neves, H. (2012). Fab Lab Kids: oficina experimental de fabricaçáo digital de brinquedos educativos. São Carlos: V!RUS.

Bonsiepe, G. (2011). Design cultura e sociedade. São Paulo: Blucher. Camargo A. C. et al. ( 2005). Robótica na periferia? Uso de tecnologias digitais na rede pública de ensino de São Paulo como ferramenta de expressão e inclusão. São Leopoldo-PR:XI Workshop de Informática em Educação. Artigo.

Cross, N. (1982). Design education for laypeople. Changing design. Chichester, UK: Wiley.

Gershenfeld,N. (2012). How to Make Almost Anything: The Digital Fabrication Revolution. US: Foreign Affairs. Artigo.

Maldonado, T. (2012). Cultura Sociedade e Técnica. São Paulo: Blucher. Mikhak,B . (2002) Fab Lab: an alternate model of ICT for development. Bangalore: ThinkCycle. artigo

Papert, S. (1980). Mindstorms: children, computers, and powerful ideas. New York: Basic Books. livro

Schön, D, Sanyal. B, \& Mitchell, W. (2001). High Technology and Low-Income Communities: Prospects for the Positive Use of Advanced Information Technology. US: The MIT Press. 